\title{
Local and systemic biomarkers in gingival crevicular fluid increase odds of periodontitis
}

Fitzsimmons TR, Sanders AE, Bartold PM, Slade GD. Local and systemic biomarkers in gingival crevicular fluid increase odds of periodontitis. J Clin Periodontol 2010; 37: 30-36. doi: 10.1111/j.1600-051X.2009.01506.x.

\begin{abstract}
Aim: To determine the independent and combined associations of interleukin- $1 \beta$ (IL-1 $\beta$ ) and C-reactive protein (CRP) in gingival crevicular fluid (GCF) on periodontitis case status in the Australian population.

Materials and Methods: GCF was collected from 939 subjects selected from the 2004-2006 Australian National Survey of Adult Oral Health: 430 cases had examinerdiagnosed periodontitis, and 509 controls did not. IL-1 $\beta$ and CRP in GCF were detected by enzyme-linked immunosorbent assays. Odds ratios (OR) and 95\% confidence intervals (CIs) were calculated in bivariate and stratified analysis and fully adjusted ORs were estimated using multivariate logistic regression.

Results: Greater odds of having periodontitis was associated with higher amounts of IL-1 $\beta(\mathrm{OR}=2.4,95 \% \mathrm{CI}=1.7-3.4$ for highest tertile of $\mathrm{IL}-1 \beta$ relative to lowest tertile $)$ and $\mathrm{CRP}(\mathrm{OR}=1.9,95 \% \mathrm{CI}=1.5-2.5$ for detectable $\mathrm{CRP}$ relative to undetectable CRP). In stratified analysis, there was no significant interaction between biomarkers $(p=0.68)$. In the multivariate analyses that controlled for conventional periodontal risk factors, these relationships remained $(\mathrm{IL}-1 \beta \mathrm{OR}=1.8,95 \% \mathrm{CI}=$ 1.1-2.6; CRP OR $=1.7,95 \% \mathrm{CI}=1.3-2.3$ ).

Conclusions: Elevated odds of clinical periodontitis was associated independently with each biomarker. This suggests that people with elevated biomarkers indicative of either local (IL-1 $\beta$ ) or systemic (CRP) inflammation are more likely to suffer from periodontal disease.
\end{abstract}

Tracy R. Fitzsimmons ${ }^{1}$, Anne E. Sanders ${ }^{2}$, P. Mark Bartold ${ }^{1}$ and Gary D. Slade ${ }^{2}$

${ }^{1}$ Centre for Orofacial Research and Learning, Colgate Australia Clinical Dental Research Centre, School of Dentistry, University of Adelaide, Adelaide, SA, Australia;

${ }^{2}$ Australian Research Centre for Population Oral Health, School of Dentistry, University of Adelaide, Adelaide, SA, Australia

Key words: C-reactive protein; gingival crevicular fluid; interleukin-1 $\beta$; periodontitis

Accepted for publication 14 October 2009
Numerous studies have shown that biomarkers of inflammatory response are elevated in people with periodontitis compared with healthy controls (Mogi et al. 1999, Gamonal et al. 2000, Engeb-

\section{Conflict of interest and source of funding statement}

The authors declare that there are no conflicts of interest in this study.

This study was supported by grants from the National Health and Medical Research Council (Project Grants \#349514 and \#299060). Dr. Sanders is supported by a NHMRC Sidney Sax Public Health Fellowship (\#399222). retson et al. 2002, Bostanci et al. 2007, Zhong et al. 2007). These biomarkers include cytokines known to be produced locally in periodontal tissues [e.g. interleukin-1 $\beta$ (IL-1 $\beta)$ ] as well as acute phase reactants that predominantly are produced in tissues remote from the infection [e.g. C-reactive protein (CRP), predominantly produced in liver]. Our own casecontrol study confirmed these findings in samples of gingival crevicular fluid (GCF) collected from a representative population-based sample of Australian adults in which we measured IL- $1 \beta$ and CRP (Fitzsimmons et al. 2009).

It is possible that inflammatory biomarkers produced in tissues remote from the periodontium become elevated in response to periodontitis only as a consequence of locally produced biomarkers in the periodontium. If that were so, then the association between CRP and periodontitis would be dependent upon IL-1 $\beta$ levels in the periodontium. However, if both CRP and IL-1 $\beta$ are independently associated with periodontitis, it would suggest the relationship is more complex, and potentially could offer insight into separate mechanisms by which local and systemic inflammatory responses are related to the disease.

The relationship between inflammatory biomarkers and periodontitis is 
most likely bidirectional. On the one hand, bacterial infection in the periodontal tissues stimulates a local immune response involving the production and release of several inflammatory mediators into the surrounding tissue. The role of one such mediator, IL- $1 \beta$, is well known in periodontal disease progression (Lo et al. 1999, Graves \& Cochran 2003) and as an indicator of active inflammation. Produced locally at the site of infection by a number of cells involved in host defence against periodontal pathogens including macrophages, neutrophils and gingival fibroblasts (Takahashi et al. 1995, Lo et al. 1999, Steffen et al. 2000), IL-1 $\beta$ is initially released to combat infection. However, persistent infection leads to the dysregulation of the immune system and a cascade of events, initiated by IL$1 \beta$, results in chronic inflammation of the periodontal supporting tissues, culminating in destruction of alveolar bone mediated via IL- $1 \beta$ regulation of osteoclasts (Graves \& Cochran 2003). These inflammatory processes in periodontal tissues can trigger a more generalized systemic response in tissues remote from the periodontium, such as the liver.

Meanwhile, inflammatory processes elsewhere in the body can contribute to periodontitis. Some systemic diseases (such as diabetes) that increase risk of periodontitis also contribute to inflammatory responses in numerous tissues, increasing systemic levels of each biomarker. Finally, there is speculation that a common underlying hyper-inflammatory phenotype might predispose people, both to periodontitis and systemic inflammation (Offenbacher \& Beck 2005). These bidirectional effects mean that elevated levels of inflammatory biomarkers may signify both the causes and consequences of periodontitis.

Given the likely bidirectional causal pathways linking periodontitis and inflammatory biomarkers, it is informative to examine these relationships using periodontitis both as an explanatory variable and separately as a dependent variable. In our previous study, we used periodontitis as an explanatory variable to show that both IL- $1 \beta$ and CRP in GCF were elevated in periodontitis cases relative to non-cases (Fitzsimmons et al. 2009). However, we did not attempt to determine if elevated levels of CRP was associated with periodontitis independently of the levels of IL- $1 \beta$.
Consequently, in the current analyses, we focus on periodontitis as the dependent variable with the primary aim to determine the combined effects of each biomarker on occurrence of clinical periodontitis. Our hypothesis was that each biomarker would contribute independently to the odds of periodontitis, and that their effects would be observable even after adjusting for conventional risk factors for periodontitis. Such a finding is consistent with the notion that both local and systemic inflammatory processes contribute to the risk of having periodontitis.

If this hypothesis is supported, findings will build on existing evidence to provide "proof-of-principle', support for a potential etiological association between both sources of inflammation and periodontitis case status.

\section{Materials and Methods Study design and sampling}

The current case-control study as previously described (Fitzsimmons et al. 2009) was nested within the National Survey of Adult Oral Health (20042006) (NSAOH) (Slade et al. 2007). The University of Adelaide's Human Research Ethics Committee granted approval for the National Survey. During the survey, 5505 people underwent standardized oral epidemiological examinations. All examined subjects provided written informed consent before the examination. Examinations included measurements of periodontal pocket depth (PD) and gingival recession on all teeth except third molars. Three sites were assessed per tooth: mesiobuccal, mid-buccal and distobuccal. Clinical periodontal attachment level was calculated as the sum of PD and recession at each site during subsequent data management. Following probing measurements, up to four periodontal sites were selected at random from among all measured periodontal sites and used for collection of GCF samples. An algorithm in the computer program recording dental examination data selected four periodontal sites at random for collection of GCF samples. Sites were selected from among all periodontally measured sites within each person's mouth, yielding up to two sites with probing depths of at least $4 \mathrm{~mm}$ and the remaining sites with shallower probing depths. GCF was collected at four sites where recession and probing depths were recorded. Where all probing depth measurements were $<4 \mathrm{~mm}$, a simple random sampling method was used to select all four sites. Otherwise, a stratified random sampling method was used to collect GCF from up to two sites with probing depth $4 \mathrm{~mm}$, and the remaining samples from sites with probing depth $<4 \mathrm{~mm}$.

\section{Case definition}

For this study, 939 examined survey participants were selected using a case-control study design. Cases were people with two or more interproximal sites with $\geqslant 4 \mathrm{~mm}$ clinical attachment loss and two or more interproximal sites with $\mathrm{PD}$ of $\geqslant 5 \mathrm{~mm}$ or more were defined as moderate or severe periodontitis cases, while non-cases had less extensive disease (Page \& Eke 2007). All examined NSAOH subjects who met criteria as periodontal cases were included in this study, while $\mathrm{NSAOH}$ subjects who were non-cases were sampled at random for this study to select an approximately equivalent number of non-cases for this study. Non-cases from the NSAOH subjects were selected at random using a random number generator in SAS (SAS v.9.2, SAS Institute, Cary, NC, USA), with frequency matching within states/territories to achieve similar numbers of cases and non-cases within each jurisdiction.

\section{GCF collection and analysis}

GCF was collected on two PerioPaper ${ }^{\mathrm{TM}}$ strips (Oraflow Inc., Plainview, NY, USA) per site as previously described (Fitzsimmons et al. 2009). Briefly, the area was isolated from saliva with cotton rolls and air dried before placing the strip into the gingival crevice for $10 \mathrm{~s}$. Paper strips were wrapped in foil before shipping to the laboratory by mail where they were frozen at $-20^{\circ} \mathrm{C}$ until processed. GCF samples contaminated with blood were processed but excluded from analysis. GCF was eluted from each individual PerioPaper ${ }^{\mathrm{TM}}$ strip as previously described (Fitzsimmons et al. 2009). GCF was eluted from Perio Paper $^{\mathrm{TM}}$ strips by a two-step centrifugation method. Each strip was eluted separately by adding elution buffer ( $1 \%$ bovine serum albumin solution, R\&D Systems, Minneapolis, MN, USA) to the strip and incubating at room temperature for $15 \mathrm{~min}$. before 
centrifugation at $3000 \mathrm{~g}$ for $5 \mathrm{~min}$. at $4^{\circ} \mathrm{C}$. This process was repeated before combining eluted solution (final volume $480 \mu \mathrm{l})$ from each pair of strips before storing at $-20^{\circ} \mathrm{C}$ until further analysis by ELISA.

Levels of IL- $1 \beta$ and CRP in GCF samples were determined using commercially available ELISA kits [R\&D Systems (IL-1 $\beta$ ) and Bender MedSystems, Vienna, Austria (CRP)] following the manufacturer's guidelines. All standards and samples were assayed in duplicate with biomarker concentrations determined from standard curves generated with $\mathrm{KC} 4$ software (BioTek Instruments, Winooski, VT, USA) using a four-parameter logistic curve-fit (IL$1 \beta)$ or linear regression (CRP) analysis.

\section{Quantifying inflammatory biomarkers}

The eluted IL- $1 \beta$ was multiplied by 0.48 to yield the amount in picograms at each periodontal site, and its natural log was computed to overcome the skewed nature of its distribution. For each person, the mean value of log (amount) was computed from among all sites assayed. For the purpose of these analyses this continuous variable was split into approximately equal-sized tertiles labelled "Low', (a mean of $1.0 \mathrm{pg}$ or less of eluted IL- $1 \beta$ among assayed sites), "Moderate" (a mean of $>1.0 \mathrm{pg}$ to $<3.3 \mathrm{pg}$ of eluted IL- $1 \beta$ ) and "High"' (a mean of $3.3 \mathrm{pg}$ or more).

The lower limit of detection of the CRP ELISA was $3 \mathrm{pg} / \mathrm{ml}$. The eluted concentration of CRP fell below the limit of detection for $58 \%$ of sites, so the data were dichotomized to indicate whether or not an amount of three or more picograms per millilitre was detected. A person-level variable was computed to indicate whether or not the study subject had detectable CRP at this threshold.

\section{Self-reported socio-demographics and medical history}

Self-reported information about demographics, smoking history, self-rated health and medical conditions were asked either during a telephone interview conducted before the examinations or using a questionnaire completed on the day of the examination. For each medical condition, subjects were asked "Has a doctor or other health professional ever told you that you have ..." (e.g. high blood pressure) and response categories were "yes", "no" and "don't know". The latter were coded as missing and not used in this analysis.

\section{Statistical analysis}

To test the study hypothesis that IL- $1 \beta$ and CRP were significant independent predictors of periodontitis, unconditional logistic regression was used to compute unadjusted odds ratios (OR) and corresponding $95 \%$ confidence intervals $(95 \%$ CIs) for these associations. ORs with 95\% CIs that excluded 1.0 were judged to be statistically significant. Odds of periodontitis were also examined for selected subject socio-demographic characteristics, cigarette smoking and self-reported history of chronic conditions, each of which is a recognized periodontal risk indicator. The variable hypothesized to modify the association between CRP and periodontitis was IL$1 \beta$. Consequently to test for potential interaction, the association between CRP and periodontitis was computed within each stratum of IL- $1 \beta$ (low, moderate, high). Crude ORs were compared with these stratum-specific estimates using the Breslow-Day test for homogeneity of the odds. Finally, significant predictors were entered into a multivariate logistic regression model to determine whether, and to what extent, elevated biomarkers remained significant periodontitis risk predictors after adjustment for conventional risk indicators. To investigate the sensitivity of these findings to the threshold used to define periodontal case status, we repeated the logistic regression analysis, excluding subjects with moderate periodontitis, to model the odds of having severe periodontitis. All analyses were conducted using SAS v.9.2 (SAS Institute).

\section{Results}

Of the 939 subjects selected from the 2004-2006 Australian National Survey of Adult Oral Health, 430 were examiner-diagnosed periodontitis cases, and the remaining 509 non-cases. In unadjusted analysis (Table 1) a doseresponse effect was evident of increasing odds of periodontitis at elevated levels of IL-1 $\beta$. Likewise subjects with detectable CRP were almost twice as likely to be cases as those with undetectable levels of this biomarker (OR 1.9, 95\% CI 1.5-2.5). Confirming previous findings, odds of periodontitis were elevated at older age and among males, non-Australian born subjects born and former and current smokers relative to their respective reference groups. Also presenting as a significant risk indicator was history of chronic health conditions. Elevated levels of IL-1 $\beta$ were found in subjects with detectable CRP and among males, the non-Australian born and diabetics, relative to females, Australian-born subjects and those with no diagnosis of diabetes. Detectable levels of CRP were found in significantly higher proportions of the aged and medically compromised than in the young and healthy, although the association with a history of high blood cholesterol was non-significant.

A non-significant statistic for the Breslow-Day test $(p=0.68)$ was evidence for no effect modification by IL$1 \beta$ levels in the association between CRP and periodontitis (Table 2). Hence no interaction term was entered into the multivariable logistic regression model.

After adjustment for covariates (Table 3), IL-1 $\beta$ and detectable CRP remained significantly associated with periodontitis risk. Furthermore, odds of periodontitis were elevated $80 \%$ for each additional decade of life lived. Males and non-Australian born subjects remained about twice as likely to be a case and current smokers had odds elevated 3.6 -fold $(95 \% \mathrm{CI}=2.4-5.5)$ relative to subjects who had never smoked.

When the odds of severe periodontitis was evaluated in a logistic regression model that excluded cases of moderate periodontitis, both mediators remained statistically significant. There was a similar magnitude of effect for IL- $1 \beta$ (for the mid-tertile of IL- $1 \beta, \mathrm{OR}=1.5$, $95 \% \mathrm{CI}=0.7-2.9$; for the high tertile of $\mathrm{IL}-1 \beta, \quad \mathrm{OR}=1.9,95 \% \quad \mathrm{CI}=1.0-3.6)$, and a slightly stronger effect for CRP $(\mathrm{OR}=3.3,95 \% \mathrm{CI}=2.0-5.5)$. The covariates listed in Table 3 also remained statistically significant, with similar or greater ORs [e.g. up to 7.8 (95\% $\mathrm{CI}=4.0-15.1)$ for current smoking]

\section{Discussion}

This study investigated the combined effects of IL- $1 \beta$ and CRP in GCF on presence of periodontitis in the Australian population. Increased odds of clinical periodontitis was associated independently with elevated biomarkers that are indicative of local (IL-1 $\beta$ ) or 
Table 1. Influence of biomarker presence in GCF on odds of periodontitis

\begin{tabular}{|c|c|c|c|c|c|c|c|}
\hline \multirow[t]{2}{*}{ Predictor } & \multirow[t]{2}{*}{ Number of subjects } & \multirow[t]{2}{*}{ Percentage with periodontitis } & \multirow[t]{2}{*}{ OR $\left(95 \% \mathrm{CI}^{*}\right)$} & \multicolumn{3}{|c|}{$\mathrm{IL}-1 \beta^{\dagger}$} & \multirow{2}{*}{$\begin{array}{c}\mathrm{CRP}^{\ddagger} \\
\text { Detectable }\end{array}$} \\
\hline & & & & Low & Medium & High & \\
\hline \multicolumn{8}{|l|}{$\mathrm{IL}-1 \beta$} \\
\hline Low-tertile & 267 & 33.3 & Ref. $^{\S}$ & - & - & - & 25.1 \\
\hline Mid-tertile & 322 & 46.3 & $1.7(1.2-2.4)$ & - & - & - & 40.7 \\
\hline High-tertile & 350 & 54.9 & $2.4(1.7-3.4)$ & - & - & - & 55.4 \\
\hline$p$-value & & & & & & & $<0.01$ \\
\hline \multicolumn{8}{|l|}{ CRP } \\
\hline Undetectable & 547 & 39.1 & Ref. & 36.6 & 34.9 & 28.5 & - \\
\hline Detectable & 392 & 55.1 & $1.9(1.5-2.5)$ & 17.1 & 33.4 & 49.5 & - \\
\hline$p$-value & & & & & $<0.01$ & & \\
\hline \multicolumn{8}{|l|}{ Age (years) } \\
\hline $15-44$ & 306 & 22.6 & Ref. & 33.7 & 33.0 & 33.3 & 35.0 \\
\hline $45-59$ & 318 & 50.3 & $3.5(2.5-4.9)$ & 27.7 & 33.3 & 40.0 & 42.8 \\
\hline $60+$ & 315 & 63.8 & $6.1(4.3-8.6)$ & 24.1 & 36.5 & 39.4 & 47.3 \\
\hline$p$-value & & & & & 0.11 & & $<0.01$ \\
\hline \multicolumn{8}{|l|}{ Gender } \\
\hline Female & 542 & 37.3 & Ref. & 30.3 & 36.2 & 33.6 & 42.4 \\
\hline Male & 397 & 57.4 & $2.3(1.7-3.0)$ & 25.9 & 31.7 & 42.3 & 40.8 \\
\hline$p$-value & & & & & 0.02 & & 0.62 \\
\hline \multicolumn{8}{|l|}{ Country of birth } \\
\hline Australia & 692 & 39.7 & Ref. & 30.9 & 35.7 & 33.4 & 41.8 \\
\hline Elsewhere & 247 & 62.8 & $2.6(1.9-3.4)$ & 21.5 & 30.4 & 48.2 & 41.7 \\
\hline$p$-value & & & & & $<0.01$ & & 0.99 \\
\hline \multicolumn{8}{|l|}{ Smoking status } \\
\hline Never & 457 & 37.9 & Ref. & 29.8 & 35.2 & 35.0 & 39.8 \\
\hline Former & 307 & 50.5 & $1.7(1.2-2.2)$ & 28.0 & 34.9 & 37.1 & 44.3 \\
\hline Current & 175 & 58.3 & $2.3(1.6-3.3)$ & 25.7 & 30.9 & 43.4 & 42.3 \\
\hline$p$-value & & & & & 0.41 & & 0.46 \\
\hline \multicolumn{8}{|l|}{ Diabetes history } \\
\hline No & 879 & 44.7 & Ref. & 29.6 & 34.5 & 36.2 & 40.7 \\
\hline Yes & 60 & 61.7 & $2.0(1.2-3.4)$ & 15.0 & 31.7 & 53.3 & 56.7 \\
\hline$p$-value & & & & & 0.01 & & 0.02 \\
\hline \multicolumn{8}{|c|}{ Cardiovascular disease history } \\
\hline No & 898 & 44.9 & Ref. & 28.8 & 34.6 & 36.5 & 41.0 \\
\hline Yes & 37 & 70.3 & $2.9(1.4-5.9)$ & 16.2 & 27.0 & 56.8 & 62.2 \\
\hline$p$-value & & & & & 0.04 & & 0.01 \\
\hline \multicolumn{8}{|l|}{ High blood pressure } \\
\hline No & 674 & 42.0 & Ref. & 29.4 & 34.1 & 36.5 & 37.2 \\
\hline Yes & 251 & 57.4 & $1.9(1.4-2.5)$ & 25.9 & 35.1 & 39.0 & 53.8 \\
\hline$p$-value & & & & & 0.56 & & $<0.01$ \\
\hline \multicolumn{8}{|l|}{ High blood cholesterol } \\
\hline No & 649 & 41.5 & Ref. & 28.8 & 36.1 & 35.1 & 40.8 \\
\hline Yes & 266 & 57.1 & $1.9(1.4-2.5)$ & 25.9 & 31.2 & 42.9 & 42.9 \\
\hline$p$-value & & & & & 0.09 & & 0.57 \\
\hline \multicolumn{8}{|l|}{ General health } \\
\hline Good to excellent & 825 & 44.0 & Ref. & 28.9 & 34.6 & 36.6 & 40.5 \\
\hline Poor to fair & 114 & 58.7 & $1.8(1.2-2.7)$ & 25.4 & 32.5 & 42.1 & 50.9 \\
\hline$p$-value & & & & & 0.51 & & 0.03 \\
\hline
\end{tabular}

*95\% CI for OR.

${ }^{\dagger}$ Percentage of people with low $(<1.0 \mathrm{pg})$, medium $(1.0$ to $<3.3 \mathrm{pg})$ and high $(\geqslant 3.3 \mathrm{pg})$ amounts of IL-1 $\beta$ eluted from GCF.

${ }^{\ddagger}$ Percentage of people with detectable CRP in GCF.

${ }^{\S}$ Reference group for OR.

CI, confidence interval; OR, odds ratio; IL-1 $\beta$, interleukin-1 $\beta$; CRP, C-reactive protein; GCF, gingival crevicular fluid.

systemic (CRP) inflammation. In addition a number of socio-demographic variables were also associated with increased odds of having periodontitis and these included age, gender, smoking status and country of birth. The findings generally were similar when the more stringent case definition of severe periodontitis was used.
These findings are consistent with those from a previous study, which demonstrated that increased levels of both IL- $1 \beta$ and CRP in GCF were associated with age and country of birth (Fitzsimmons et al. 2009). In addition to these associations, the present study determined that the levels of IL- $1 \beta$ and the presence of CRP in GCF were sig- nificantly associated with a history of diabetes or cardiovascular disease. Furthermore, detectable levels of CRP in GCF were also influenced by a history of high blood pressure and selfreported poor health. These findings strengthen the notion that periodontitis involves inflammatory responses that are associated with local and systemic 
Table 2. Odds ratios (OR) and corresponding 95\% confidence intervals (CI) between C-reactive protein and periodontitis case status, stratified by levels of Interleukin- $1 \beta$

\begin{tabular}{llccc}
\hline $\begin{array}{l}\text { Interleukin-1 } \beta \\
\text { (tertiles of amount) }\end{array}$ & $\begin{array}{c}\text { C-reactive } \\
\text { protein }\end{array}$ & $\begin{array}{c}\text { Number of } \\
\text { subjects }\end{array}$ & $\begin{array}{c}\text { Periodontitis } \\
\text { cases (\%) }\end{array}$ & OR (95\% CI) \\
\hline Low & Undetectable & 200 & 31.5 & Ref.* \\
& Detectable & 67 & 38.8 & $1.4(0.8-2.5)$ \\
Medium & Undetectable & 191 & 41.2 & Ref. \\
& Detectable & 131 & 53.4 & $1.6(1.0-2.5)$ \\
High & Undetectable & 156 & 46.1 & Ref. \\
& Detectable & 194 & 61.9 & $1.9(1.2-2.9)$ \\
\hline
\end{tabular}

*Reference group for OR.

$\chi^{2}(2 \mathrm{df})=0.68, p=0.77$ from Breslow-Day test of homogeneity of odds ratios among three tertiles of IL- $1 \beta$.

Table 3. Multivariate logistic regression model predicting odds of periodontitis $(n=938)$

\begin{tabular}{lc}
\hline Predictor & Odds ratio $(95 \%$ confidence interval $)$ \\
\hline Interleukin-1 $\beta$ & Ref. $^{*}$ \\
Low & $1.6(1.1-2.6)$ \\
Mid & $1.8(1.2-2.6)$ \\
High & Ref. \\
C-reactive protein & $1.7(1.3-2.3)$ \\
$\quad$ Undetectable & \\
Detectable & $1.8(1.6-2.0)$ \\
Age & \\
Per 10 year increment & Ref. \\
Gender & $1.9(1.4-2.6)$ \\
Female & Ref. \\
Male & $1.3(1.0-1.9)$ \\
Smoking & $3.6(2.4-5.5)$ \\
Never & \\
Former & Ref. \\
Current & $2.2(1.5-3.1)$ \\
Country of birth & \\
Australia & \\
Elsewhere & \\
\hline
\end{tabular}

*Reference group for odds ratio.

patterns of inflammation and that several systemic inflammatory conditions may impact on periodontitis status patterns. Although this study cannot identify the tissues in which each mediator was produced, it seems likely that CRP appears in GCF primarily as a serum exudate following its production in the liver. This is consistent with other studies demonstrating that oral pathogens elicit an immune response with elevated serum levels of CRP (Noack et al. 2001) and IL-1 $\beta$ (Swoboda et al. 2008). While it is well accepted that local biomarkers, including various cytokines, and acute phase proteins, are present in GCF (Armitage 1996) the identification and measurement of CRP in GCF has been relatively limited. Besides our previous study (Fitzsimmons et al. 2009), there are few other reports documenting the presence of CRP in GCF (Sibraa et al. 1991, Tuter et al. 2007).
While increased levels of CRP in GCF of periodontitis patients noted in the present study are consistent with previous reports of increased CRP in serum from subjects with periodontitis (Slade et al. 2000, Glurich et al. 2002, Salzberg et al. 2006), we cannot comment on the clinical significance, if any, of CRP thresholds used in this analysis. Because there are no previous studies showing clinical significance of CRP thresholds in GCF, we used the assay's detection threshold of $3 \mathrm{pg} / \mathrm{ml}$ in eluted GCF because it identified sufficient number of subjects to permit analysis of associations. In a study of 67 subjects (including 20 periodontal patients), Tuter et al. (2007) analysed CRP in serum and GCF, and found only a weak correlation (Pearson's $r=-0.027, p>0.05$ ). Additional studies, preferably using longitudinal designs that predicted risk of periodontitis or other diseases, would be needed to determine the clinical significance of this threshold.

The presence of CRP in GCF in the current study also builds on the growing body of evidence supporting a relationship between periodontal disease and systemic inflammatory conditions such as diabetes, cardiovascular disease and rheumatoid arthritis (Mercado et al. 2001, Persson et al. 2003, Perrino 2007). Associations between these conditions and periodontitis are most likely mediated by increased inflammatory burden and the induction of acute phase proteins such as CRP (D'Aiuto et al. 2004, Joshipura et al. 2004, Bizzarro et al. 2007, Abou-Raya et al. 2008). Of these associations, one of the most significant appears to be the increase in CRP levels in people with periodontitis. As demonstrated by us and others, this has been implicated as a risk factor for future cardiovascular events (Wu et al. 2000, D'Aiuto et al. 2004, Sun et al. 2009).

While there is accumulating evidence implicating periodontitis as a risk factor for other chronic conditions, the bidirectional nature of these associations needs to be recognized and analysed. For example, in our study the odds of having periodontitis was increased approximately 2.5 by $\mathrm{IL}-1 \beta$ and two times by detectable CRP in unadjusted analysis. Adjusted ORs were slightly lower, with high levels of IL- $1 \beta$ and detectable CRP increasing the odds of having periodontitis by 1.8 and 1.7 times, respectively. These data are similar to those reported by Linden et al (2008) where the adjusted OR of periodontitis in a multivariable analysis was increased by increased serum levels of CRP to $2.49(95 \% \mathrm{CI}=1.16-5.35)$. The slight difference in values reported in these two studies may be explained by serum versus GCF detection of CRP and varying differences in periodontitis case definitions. Irrespective of these differences it is apparent that reasons for increased levels of biomarkers in GCF may be the result of periodontal disease or they may be a consequence of other systemic conditions such as cardiovascular disease diabetes, or rheumatoid arthritis. This important distinction remains to be elucidated (Grossi \& Genco 1998, Mealey 2006).

An important finding of the present study was that although the stratified analysis of the presence of IL- $1 \beta$ together with CRP showed a trend towards an increased risk of having 
periodontitis, there was no significant interaction between the two biomarkers. The notion that cytokines and immune regulators influence the risk of systemic disease is not a new concept. The combination of IL- 6 and IL- $1 \beta$ independently increases the risk of diabetes by approximately three times (Spranger et al. 2003). Increased CRP and IL-6 are also shown to increases the risk of diabetes type 2 by 2.3 and 4.2 times, respectively (Pradhan et al. 2001). Given that IL- $1 \beta$ and CRP are part of a much larger network of cytokines and inflammatory mediators, analysis of the combination of these two biomarkers in isolation may partially explain these results. Other cytokines, particularly tumour necrosis factor- $\alpha$ and IL-6 are two key regulators of IL- $1 \beta$ and CRP, respectively. In addition, the induction of CRP expression by IL-6 is synergistic with IL-1 $\beta$ (Ganapathi et al. 1991). Well-documented evidence of increased levels of IL-6 in GCF from subjects with periodontitis (Mogi et al. 1999, Sun et al. 2009) may partially explain the present observations of CRP in GCF.

Aside from the limitations of a casecontrol study design, which preclude us from determining whether inflammatory mediators are a cause or consequence of periodontitis (or both), the principal limitations of this study arise from simplification of measurements that are needed for population studies. Specifically, we assessed GCF at only four sites per subject, and clinical periodontal measurements were made at only three sites per tooth, not six. In addition, we were reliant on self-reported information about medical history and health behaviours (e.g. smoking) that are known to influence inflammation. Nonetheless, the selection of cases and controls from a larger population survey helps to minimize biases association with sampling from patient groups, and it means that the findings can be generalized to the Australian adult population.

In summary, the odds of individuals having periodontitis were increased by the presence of biomarkers traditionally indicative of local (IL-1 $\beta$ ) and systemic inflammation (CRP). These findings support our hypothesis that people with evidence of local or systemic inflammation are more likely to suffer from periodontal disease. Importantly, the effects of IL- $1 \beta$ and CRP were noted to be independent and statistically significant in a multivariable model that adjusted for conventional risk indicators. Hence we conclude that there was no interaction between IL- $1 \beta$ and CRP. Such independence adds further to our understanding that a chronic disease such as periodontitis may be under bidirectional influences. For example, a local inflammatory response could be expected to result in a local increase in production of inflammatory cytokines such as IL-1 $\beta$. However, localized inflammation can also result in a more generalized systemic inflammatory response in distant organs, including the liver. In addition, individuals who manifest a hyper-inflammatory phenotype are predisposed to tissue destruction in response to local periodontal infection. Thus local and systemic factors may act both independently and synergistically in a complex disease such as periodontitis. In order to address these issues a prospective cohort study is required. Overall, these data support our original hypothesis that people with evidence of local or systemic inflammatory biomarkers in GCF are more likely to suffer from periodontal disease.

\section{References}

Abou-Raya, S., Abou-Raya, A., Naim, A. \& Abuelkheir, H. (2008) Rheumatoid arthritis, periodontal disease and coronary artery disease. Clinical Rheumatology 27, 421-427.

Armitage, G. C. (1996) Periodontal diseases: diagnosis. Annals of Periodontology 1, 37215.

Bizzarro, S., van der Velden, U., ten Heggeler, J. M., Leivadaros, E., Hoek, F. J., Gerdes, V. E., Bakker, S. J., Gans, R. O., Ten Cate, H. \& Loos, B. G. (2007) Periodontitis is characterized by elevated PAI-1 activity. Journal of Clinical Periodontology 34, 574-580.

Bostanci, N., Ilgenli, T., Emingil, G., Afacan, B., Han, B., Toz, H., Atilla, G., Hughes, F. J. \& Belibasakis, G. N. (2007) Gingival crevicular fluid levels of RANKL and OPG in periodontal diseases: implications of their relative ratio. Journal of Clinical Periodontology 34, 370-376.

D’Aiuto, F., Ready, D. \& Tonetti, M. S. (2004) Periodontal disease and C-reactive proteinassociated cardiovascular risk. Journal of Periodontal Research 39, 236-241.

Engebretson, S. P., Grbic, J. T., Singer, R. \& Lamster, I. B. (2002) GCF IL-1beta profiles in periodontal disease. Journal of Clinical Periodontology 29, 48-53.

Fitzsimmons, T. R., Sanders, A. E., Slade, G. D. \& Bartold, P. M. (2009) Biomarkers of periodontal inflammation in the Australian adult population. Australian Dental Journal 54, 115-122.
Gamonal, J., Acevedo, A., Bascones, A., Jorge, O. \& Silva, A. (2000) Levels of interleukin-1 beta, -8 , and -10 and RANTES in gingival crevicular fluid and cell populations in adult periodontitis patients and the effect of periodontal treatment. Journal of Periodontology 71, 1535-1545.

Ganapathi, M. K., Rzewnicki, D., Samols, D., Jiang, S. L. \& Kushner, I. (1991) Effect of combinations of cytokines and hormones on synthesis of serum amyloid A and C-reactive protein in Hep 3B cells. Journal of Immunology 147, 1261-1265.

Glurich, I., Grossi, S., Albini, B., Ho, A., Shah, R., Zeid, M., Baumann, H., Genco, R. J. \& De Nardin, E. (2002) Systemic inflammation in cardiovascular and periodontal disease: comparative study. Clinical and Diagnostic Laboratory Immunology 9, 425-432.

Graves, D. T. \& Cochran, D. (2003) The contribution of interleukin-1 and tumor necrosis factor to periodontal tissue destruction. Journal of Periodontology 74, 391-401.

Grossi, S. G. \& Genco, R. J. (1998) Periodontal disease and diabetes mellitus: a two-way relationship. Annals of Periodontology 3, 51-61.

Joshipura, K. J., Wand, H. C., Merchant, A. T. \& Rimm, E. B. (2004) Periodontal disease and biomarkers related to cardiovascular disease. Journal of Dental Research 83, 151155.

Linden, G. J., McClean, K., Young, I., Evans, A. \& Kee, F. (2008) Persistently raised Creactive protein levels are associated with advanced periodontal disease. Journal of Clinical Periodontology 35, 741-747.

Lo, Y. J., Liu, C. M., Wong, M. Y., Hou, L. T. \& Chang, W. K. (1999) Interleukin 1betasecreting cells in inflamed gingival tissue of adult periodontitis patients. Cytokine 11, 626-633.

Mealey, B. L. (2006) Periodontal disease and diabetes: a two-way street. Journal of the American Dental Association 137 (Suppl.), 26S-31S.

Mercado, F. B., Marshall, R. I., Klestov, A. C. \& Bartold, P. M. (2001) Relationship between rheumatoid arthritis and periodontitis. Journal of Periodontology 72, 779 787.

Mogi, M., Otogoto, J., Ota, N., Inagaki, H., Minami, M. \& Kojima, K. (1999) Interleukin 1 beta, interleukin 6 , beta 2-microglobulin, and transforming growth factor-alpha in gingival crevicular fluid from human periodontal disease. Archives of Oral Biology 44, 535539.

Noack, B., Genco, R. J., Trevisan, M., Grossi, S., Zambon, J. J. \& De Nardin, E. (2001) Periodontal infections contribute to elevated systemic C-reactive protein level. Journal of Periodontology 72, 1221-1227.

Offenbacher, S. \& Beck, J. D. (2005) A perspective on the potential cardioprotective benefits of periodontal therapy. American Heart Journal 149, 950-954.

Page, R. C. \& Eke, P. I. (2007) Case definitions for use in population-based surveillance of 
periodontitis. Journal of Periodontology 78, 1387-1399.

Perrino, M. A. (2007) Diabetes and periodontal disease: an example of an oral/systemic relationship. The New York State Dental Journal 73, 38-41.

Persson, R. G., Ohlsson, O., Pettersson, T. \& Renvert, S. (2003) Chronic periodontitis, a significant relationship with acute myocardial infarction. European Heart Journal 24, 2108-2115.

Pradhan, A. D., Manson, J. E., Rifai, N., Buring, J. E. \& Ridker, P. M. (2001) C-reactive protein, interleukin 6 , and risk of developing type 2 diabetes mellitus. The Journal of the American Medical Association 286, 327-334.

Salzberg, T. N., Overstreet, B. T., Rogers, J. D., Califano, J. V., Best, A. M. \& Schenkein, H. A. (2006) C-reactive protein levels in patients with aggressive periodontitis. Journal of Periodontology 77, 933-939.

Sibraa, P. D., Reinhardt, R. A., Dyer, J. K. \& DuBois, L. M. (1991) Acute-phase protein detection and quantification in gingival crevicular fluid by direct and indirect immunodot. Journal of Clinical Periodontology 18, 101-106.

Slade, G. D., Offenbacher, S., Beck, J. D., Heiss, G. \& Pankow, J. S. (2000) Acutephase inflammatory response to periodontal disease in the US population. Journal of Dental Research 79, 49-57.

\section{Clinical Relevance}

Scientific rationale for study: Increasing evidence suggests that periodontal disease consists of local and systemic inflammatory components. Most research investigating systemic biomarkers has focussed on serum concentrations. We examined the relationship between the presence of local (IL-1 $\beta$ ) and sys-
Slade, G. D., Spencer, A. J. \& Roberts-Thomson, K. F. (eds). (2007) Australia's Dental Generations: the National Survey of Adult Oral Health 2004-06, Vol. 34. Canberra: Australian Institute of Health and Welfare.

Spranger, J., Kroke, A., Mohlig, M., Hoffmann, K., Bergmann, M. M., Ristow, M., Boeing, H. \& Pfeiffer, A. F. (2003) Inflammatory cytokines and the risk to develop type 2 diabetes: results of the prospective population-based European Prospective Investigation into Cancer and Nutrition (EPIC)-Potsdam Study. Diabetes 52, 812-817.

Steffen, M. J., Holt, S. C. \& Ebersole, J. L. (2000) Porphyromonas gingivalis induction of mediator and cytokine secretion by human gingival fibroblasts. Oral Microbiology and Immunology 15, 172-180.

Sun, X. J., Meng, H. X., Shi, D., Xu, L., Zhang, L., Chen, Z. B., Feng, X. H., Lu, R. F. \& Ren, X. Y. (2009) Elevation of C-reactive protein and interleukin-6 in plasma of patients with aggressive periodontitis. Journal of Periodontal Research 44, 311-316.

Swoboda, J. R., Kiyak, H. A., Darveau, R. \& Persson, G. R. (2008) Correlates of periodontal decline and biologic markers in older adults. Journal of Periodontology 79, 1920 1926.

Takahashi, K., Poole, I. \& Kinane, D. F. (1995) Detection of interleukin-1 beta mRNAexpressing cells in human gingival crevicular

temic (CRP) biomarkers in GCF and risk of periodontitis.

Principal findings: Odds of having periodontitis were increased by presence of local and systemic biomarkers. High levels of IL-1 $\beta$ were positively associated with a history of diabetes, cardiovascular disease and high blood cholesterol. Detectable CRP was positively associated fluid by in situ hybridization. Archives of Oral Biology 40, 941-947.

Tuter, G., Kurtis, B. \& Serdar, M. (2007) Evaluation of gingival crevicular fluid and serum levels of high-sensitivity C-Reactive protein in chronic periodontitis patients with or without coronary artery disease. Journal of Periodontology 78, 2319-2324.

Wu, T., Trevisan, M., Genco, R. J., Falkner, K. L., Dorn, J. P. \& Sempos, C. T. (2000) Examination of the relation between periodontal health status and cardiovascular risk factors: serum total and high density lipoprotein cholesterol, C-reactive protein, and plasma fibrinogen. American Journal of Epidemiology 151, 273-282.

Zhong, Y., Slade, G. D., Beck, J. D. \& Offenbacher, S. (2007) Gingival crevicular fluid interleukin-1beta, prostaglandin E2 and periodontal status in a community population. Journal of Clinical Periodontology 34, 285293.

Address:

Tracy R Fitzsimmons

Centre for Orofacial Research and Learning

Colgate Australia Clinical Dental Research

Centre

2nd Floor, Adelaide Dental Hospital

Frome Road, Adelaide, SA 5005

Australia

Email: tracy.fitzsimmons@adelaide.edu.au

with a history of diabetes, cardiovascular disease and hypertension. Practical implications: Biomarkers that indicate systemic inflammation observed in periodontitis may contribute not only to periodontal disease pathogenesis but also the inflammatory load of other systemic conditions such as diabetes and cardiovascular disease. 